\title{
Measuring the elements of the optical density matrix
}

\author{
K. L. Pregnell周 and D. T. Peggif \\ School of Science, Griffith University, Brisbane 4111, Australia
}

(Dated: February 1, 2008)

\begin{abstract}
Most methods for experimentally reconstructing the quantum state of light involve determining a quasiprobability distribution such as the Wigner function. In this paper we present a scheme for measuring individual density matrix elements in the photon number state representation. Remarkably, the scheme is simple, involving two beam splitters and a reference field in a coherent state.

PACS numbers: 03.65. Wj, 42.50.-p
\end{abstract}

\section{INTRODUCTION}

It is now well established that the quantum state of light can be measured. The first experimental evidence of this [1] followed the work of Vogel and Risken [2], where it was shown that the Wigner function could be reconstructed from a complete ensemble of measured quadrature amplitude distributions. The authors of [1] measured the quadrature distributions using balanced homodyne techniques. In the case of inefficient homodyne detectors, a more general s-parameterized quasiprobability distribution is obtained resulting in a smoothed Wigner function. In either case, to obtain the quasiprobability phase space distribution from the measured data a rather complicated inverse transformation is required.

Novel techniques which avoid this transformation are aimed at measuring the quasiprobability distribution more directly. This can be achieved, for example, in unbalanced homodyne counting experiments [3, 4], where a weighted sum of photocount statistics are combined to obtain a single point in the phase space distribution. The entire distribution is then obtained by scanning the magnitude and phase of the local oscillator over the region of interest while repeating the photon counting at each point. Perhaps the most direct method of obtaining a quasiprobability distribution is to use heterodyne [5] or double homodyne 6, 7] detection techniques where the $Q$ function is measured. The $Q$ function is related to the Wigner function through a convolution with a gaussian distribution which effectively washes out many of the interesting quantum features. It is possible to recover these features by deconvoluting the $Q$ function, however this requires multiplying by an exponentially increasing function thereby introducing a crucial dependence on sampling noise [8]. Other non-tomographic state reconstruction schemes are proposed in for fields in a cavity, in 10 for trapped atoms and in 11] where use of a Schrödinger-cat state probe is suggested. Further discussion of such techniques can be found in the recent review by Welsch and Vogel [12], the book by Leonhardt

\footnotetext{
*Electronic address: K.Pregnell@mailbox.gu.edu.au
}

${ }^{\dagger}$ Electronic address: D.Pegg@sct.gu.edu.au
113 and in [14.

A different approach has been suggested by Steuernagel and Vaccaro [15], who have proposed an interesting nonrecursive scheme to measure not the quasiprobability distribution, but rather the density operator in the photon number basis. The scheme is relatively direct in that only a finite number of different measurements are required to determine each matrix element. However, the major disadvantage of this scheme is that determination of each matrix element, $\rho_{M N}$, requires the preparation of a two-state superposition of $|N\rangle$ and $|M\rangle$ for use as a probe field. Not only has the preparation of such fields not yet been achieved, but also the experiment requires a change of the probe field for the measurement of each matrix element.

In this paper we investigate an alternative nonrecursive scheme to that of Steuernagel and Vaccaro that also measures the individual density matrix elements in the photon number basis. Remarkably we find that this can be achieved with a single reference field that can be in an easily prepared coherent state. The only changes to the reference field needed to measure all the matrix elements are simple phase shifts. The integral transformation required in the tomographic technique is avoided and is replaced by a summation of only four easily measurable probabilities. We find that this technique is particulary suited for measurements of low intensity states, such as that used in [16], where it offers some simplifications over the tomographic methods.

\section{MEASUREMENT TECHNIQUE}

The density matrix element $\rho_{M N}$ in the number state representation of a density operator $\hat{\rho}$ is given by

$$
\rho_{M N}=\operatorname{Tr}(\hat{\rho}|N\rangle\langle M|) .
$$

This can be compared with the probability for an outcome event $e$ of a measurement on a system in state $\hat{\rho}$, which is given from general quantum measurement theory 17 by

$$
P(e)=\operatorname{Tr}[\hat{\rho} \widehat{\Pi}(e)]
$$

where $\widehat{\Pi}(e)$ is the element of a probability operator measure (POM) associated with the event $e$. Comparing 


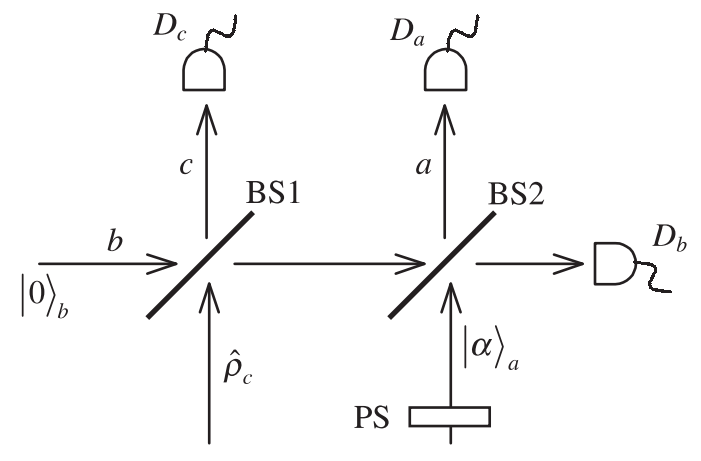

FIG. 1: Apparatus for measuring the density matrix elements of light. BS1 and BS2 are beam splitters. The field to be measured and a reference field in a coherent state are in the input modes $c$ and $a$ of BS1 and BS2 respectively. A vacuum is in the input mode $b$ and photon counters are in the output modes. Phase shifter PS adjusts the phase of the coherent state.

(2.1) and 2.2) suggests that if we could find a POM element equal to the operator $|N\rangle\langle M|$ then we could find the matrix element $\rho_{M N}$ simply by measuring the probability $P(e)$. This of course is not possible as the probability must be between zero and one but the matrix element need not even be real. However if we could synthesize the operator $|N\rangle\langle M|$ by a linear combination of different POM elements then we could find the matrix element from the same linear combination of the associated measurable probabilities. We adopt this operator synthesis approach in this paper.

The proposed measurement technique uses the arrangement shown in Fig. 1. It consists of two symmetric beam splitters labelled BS1 with input modes $b$ and $c$ and BS2 with input modes $b$ and $a$. BS2 is a 50/50 beam splitter but we keep the transmission to reflection coefficient ratio for BS1 general for now. In the output modes are photodetectors $D_{a}, D_{b}$ and $D_{c}$. The input fields in modes $b$ and $a$ are respectively in a vacuum state $|0\rangle_{b}$ and a coherent state $|\alpha\rangle_{a}$. The optimum value of the amplitude of the coherent state will be discussed later. The field to be measured, in state $\hat{\rho}_{c}$, is in the input mode $c$ of BS1. In the entry port of BS2 is a phase shifter PS capable of altering the phase of the coherent state, thereby changing the argument of the coherent state amplitude. We let the amplitude of the coherent state be $\alpha=|\alpha| \exp (i \varphi)$ at the entry of BS2, that is the argument $\varphi$ of $\alpha$ incorporates the phase shift. We let $\varphi$ be a function of two numbers $\beta$ and $j$, that is $\varphi=\varphi(\beta, j)$, that will be specified later.

For simplicity, we assume that the distance between the beam splitters is an integer number of wavelengths of the light, which allows us to ignore the evolution of the light, which is just a phase shift, between the beam splitters. If this is difficult experimentally, the discrepancy can be offset by an adjustment of the phase shifter. The complete time evolution operator is then $\widehat{R}_{2} \widehat{R}_{1}$ where $\widehat{R}_{2}$ and $\widehat{R}_{1}$ are the unitary operators for the action of beam splitters BS2 and BS1. Let $e=\left(n_{a}, n_{b}, n_{c}\right)$ be the event that photodetectors $D_{a}, D_{b}$ and $D_{c}$ register $n_{a}, n_{b}$ and $n_{c}$ photocounts respectively. The probability for this event is

$$
\begin{gathered}
P_{\beta j}(e)=\operatorname{Tr}_{a b c}\left(\widehat{R}_{2} \widehat{R}_{1} \hat{\sigma} \widehat{R}_{1}^{\dagger} \widehat{R}_{2}^{\dagger}\left|n_{a}\right\rangle_{a a}\left\langle n_{a}|\otimes| n_{b}\right\rangle_{b b}\left\langle n_{b}\right|\right. \\
\left.\otimes\left|n_{c}\right\rangle_{c c}\left\langle n_{c}\right|\right),
\end{gathered}
$$

where we have written the combined density operator for the three input fields as

$$
\hat{\sigma}=|\alpha\rangle_{a a}\langle\alpha|\otimes| 0\rangle_{b b}\langle 0| \otimes \hat{\rho}_{c}
$$

and, as the subscripts imply, the trace is over the state spaces for all three modes. The subscript $\beta j$ on the probability in (2.3) is to show explicitly that the probability is a function of the argument $\varphi(\beta, j)$ of $\alpha$, that is, it is a function of the setting of the phase shifter. Using the cyclic property of the trace we can write (2.3) as

$$
P_{\beta j}(e)=\operatorname{Tr}_{c}\left[\hat{\rho}_{c} \widehat{\Pi}_{\beta j}(e)\right],
$$

where

$$
\widehat{\Pi}_{\beta j}(e)=|q\rangle_{c c}\langle q|
$$

with

$$
|q\rangle_{c}={ }_{b}\left\langle 0\left|\widehat{R}_{1}^{\dagger}\left\langle\alpha\left|\widehat{R}_{2}^{\dagger}\right| n_{a}\right\rangle_{a}\right| n_{b}\right\rangle_{b}\left|n_{c}\right\rangle_{c} .
$$

From (2.5) we see that $\widehat{\Pi}_{\beta j}(e)$ is an element of the POM for the measuring device that comprises all of the arrangement depicted in Fig. 1 except for the field to be measured. In general the elements of $\widehat{\Pi}_{\beta j}(e)$ are not necessarily orthogonal in that $\widehat{\Pi}_{\beta j}(e) \widehat{\Pi}_{\beta j}\left(e^{\prime}\right)$ is not necessarily zero for $e \neq e^{\prime}$. The origin of the non-orthogonality is the introduction of the two reference modes $a$ and $b$. The effect of these two ancillary modes is to cube the dimensionality of the system space. In considering the measuring apparatus to consist of everything in Fig. 1 except the state to be measured, we effectively reduce the apparatus to a single mode measuring device with many more POM elements than the dimensionality of the single mode. This means that the POM elements cannot all be orthogonal to one another.

Our aim is to find a linear combination of POM elements equal to the operator $|N\rangle\langle M|$. It is convenient to write this operator as $|N\rangle\langle N+\lambda|$ and consider separately the cases where $\lambda$ is even and odd. We examine first the case where $\lambda$ is even. Consider the particular detection event $e_{1}=(\lambda / 2, \lambda / 2, N)$ in which the photodetectors $D_{a}$, $D_{b}$ and $D_{c}$ detect $\lambda / 2, \lambda / 2$ and $N$ photocounts respectively. As shown in the Appendix, this turns out to be the optimum detection event for $\lambda$ even. The unitary operator $\widehat{R}_{1}$ for BS1 is given by 18

$$
\widehat{R}_{1}=\exp \left[i \eta\left(\hat{c}^{\dagger} \hat{b}+\hat{b}^{\dagger} \hat{c}\right)\right]
$$


where $\cos \eta=t$ and $\sin \eta=r$ are the transmission and reflection coefficients of BS1. $\widehat{R}_{2}$ for the $50 / 50$ beam splitter BS2 is a similar function of $\hat{a}$ and $\hat{b}$ with $\eta=\pi / 4$. Using these expressions with $n_{a}, n_{b}$ and $n_{c}$ equal to $\lambda / 2$, $\lambda / 2$ and $N$, we find from the Appendix that (2.7) becomes

$$
|q\rangle_{c}=\sum_{n=N}^{N+\lambda} f_{n} \exp [-i(N+\lambda-n) \varphi(\beta, j)]|n\rangle_{c}
$$

where

$$
f_{n}=\frac{(2 i)^{-\lambda / 2} t^{N}|\alpha|^{\lambda+N-n}(-i r)^{n-N} \sqrt{n !}}{\exp \left(|\alpha|^{2} / 2\right)[(\lambda+N-n) / 2] ![(n-N) / 2] ! \sqrt{N !}}
$$

if $n-N$ is even and $f_{n}=0$ if $n-N$ is odd. The POM element (2.6) for the detection event $e_{1}$ then becomes

$$
\widehat{\Pi}_{\beta j}\left(e_{1}\right)=\sum_{n, m=N}^{N+\lambda} f_{n} f_{m}^{*} \exp [i(n-m) \varphi(\beta, j)]|n\rangle_{c c}\langle m| .
$$

The terms with $n-m$ odd are all zero.

For $\lambda=0$ we find from (2.11) that the POM element in (2.6) is just proportional to $|N\rangle_{c c}\langle N|$, allowing us to find the diagonal elements $\rho_{N N}$ of the density matrix from the probability of detecting the event $(0,0, N)$.

For $\lambda$ even and non-zero, we let $\varphi(\beta, j)$ take particular values

$$
\varphi(\beta, j)=\frac{\beta \pi}{\lambda}+\frac{2 \pi j}{\lambda}
$$

and consider a modified measurement procedure in which $\beta$ is held constant but the value of $j$ is cycled so that it takes all the integer values from 0 to $(\lambda / 2)-1$ with equal probability. This measurement procedure will have its own probability operator measure comprised of elements $\widehat{\Pi}_{\beta}(e)$. This will be different from our previous POM with elements $\widehat{\Pi}_{\beta j}(e)$ because it describes a different measurement process. The POM element for detecting the event $e_{1}$ by means of this cycling procedure will be given by

$$
\begin{aligned}
\widehat{\Pi}_{\beta}\left(e_{1}\right) & =\frac{2}{\lambda} \sum_{j=0}^{\lambda / 2-1} \widehat{\Pi}_{\beta j}\left(e_{1}\right) \\
= & \frac{2}{\lambda} \sum_{n, m=N}^{N+\lambda}\left\{f_{n} f_{m}^{*} \exp [i(n-m)(\beta \pi / \lambda)]\right. \\
\times & \left.\sum_{j=0}^{\lambda / 2-1} \exp [i(n-m) 2 \pi j / \lambda]|n\rangle_{c}\langle m|\right\} .
\end{aligned}
$$

The associated probability can be obtained in practice from the occurrence frequency of the event $e_{1}$ as we cycle through the values of $j$ with the experiment being repeated an equal number of times for each value of $j$. Because we need only consider terms in (2.13) for which $n-m$ is even, we can take the factor involving the summation over $j$ as zero unless $n-m$ is zero or $\pm \lambda$, in which case it equals $\lambda / 2$. This gives us

$$
\begin{aligned}
\widehat{\Pi}_{\beta}\left(e_{1}\right)= & \sum_{n=N}^{N+\lambda}\left|f_{n}\right|^{2}|n\rangle_{c c}\langle n|+\left[f_{N} f_{N+\lambda}^{*}\right. \\
& \left.\times \exp (-i \pi \beta)|N\rangle_{c c}\langle N+\lambda|+h . c .\right](2.14)
\end{aligned}
$$

By choosing different values for $\beta$, we obtain different cycling experiments, each with its own POM. Experimentally this means cycling through a different set of phase settings. It is not difficult to see from (2.14) that a linear combination of POM elements $\widehat{\Pi}_{\beta}\left(e_{1}\right)$ with $\beta$ taking the values $0,1,1 / 2$ and $3 / 2$ is required to synthesize the operator $|N\rangle\langle N+\lambda|$. Specifically,

$$
\begin{aligned}
& |N\rangle\langle N+\lambda|= \\
& \quad \frac{\widehat{\Pi}_{0}\left(e_{1}\right)-\widehat{\Pi}_{1}\left(e_{1}\right)+i\left[\widehat{\Pi}_{1 / 2}\left(e_{1}\right)-\widehat{\Pi}_{3 / 2}\left(e_{1}\right)\right]}{4 f_{N} f_{N+\lambda}^{*}}
\end{aligned}
$$

where

$$
f_{N} f_{N+\lambda}^{*}=t^{2 N}(i r / 2)^{\lambda}\left|a_{0} a_{\lambda}^{*}\right|\left(\begin{array}{c}
\lambda \\
\lambda / 2
\end{array}\right)\left(\begin{array}{c}
N+\lambda \\
N
\end{array}\right)^{1 / 2}
$$

is a normalisation constant with $a_{n}=\langle n \mid \alpha\rangle$.

Taking the trace of the product of the density operator $\hat{\rho}_{c}$ of the field to be measured with both sides of (2.15) gives the desired matrix element in terms of the measurable probabilities $P_{\beta}\left(e_{1}\right)$ for detecting the event $\left(e_{1}\right)$ :

$$
\begin{aligned}
& \left\langle N+\lambda\left|\hat{\rho}_{c}\right| N\right\rangle= \\
& \quad \frac{P_{0}\left(e_{1}\right)-P_{1}\left(e_{1}\right)+i\left[P_{1 / 2}\left(e_{1}\right)-P_{3 / 2}\left(e_{1}\right)\right]}{4 f_{N} f_{N+\lambda}^{*}}
\end{aligned}
$$

for $\lambda$ non-zero and even. The complex conjugate of 2.17) is $\left\langle N\left|\hat{\rho}_{c}\right| N+\lambda\right\rangle$.

To find the density matrix element for $\lambda$ odd, we consider the detection event $e_{2}=[(\lambda+1) / 2,(\lambda-1) / 2, N]$, which is shown in the Appendix to be the optimum detection event for this case. A derivation similar to that above eventually yields the associated POM element of the form

$$
\widehat{\Pi}_{\beta j}\left(e_{2}\right)=\sum_{n, m=N}^{N+\lambda} g_{n} g_{m}^{*} \exp [i(n-m) \varphi(\beta, j)]|n\rangle_{c c}\langle m| .
$$

In (2.18) $n-m$ takes all integer values from $+\lambda$ to $-\lambda$. We consider a measurement procedure where $\beta$ is held constant but $j$ takes all values from 0 to $\lambda-1$ with equal probability. The POM element $\widehat{\Pi}_{\beta}\left(e_{2}\right)$ for detecting the event $e_{2}$ with this procedure will be given by

$$
\widehat{\Pi}_{\beta}\left(e_{2}\right)=\frac{1}{\lambda} \sum_{j=0}^{\lambda-1} \widehat{\Pi}_{\beta j}\left(e_{2}\right)
$$




$$
\begin{aligned}
& =\frac{1}{\lambda} \sum_{n, m=N}^{N+\lambda}\left\{g_{n} g_{m}^{*} \exp [i(n-m)(\beta \pi / \lambda)]\right. \\
& \left.\times \sum_{j=0}^{\lambda-1} \exp [i(n-m) 2 \pi j / \lambda]|n\rangle_{c c}\langle m|\right\} .
\end{aligned}
$$

The factor involving the summation over $j$ is zero unless $n-m=0$ or $\pm \lambda$ and is then equal to $\lambda$. We find that the formula for the density matrix element in terms of the measurable probabilities $P_{\beta}\left(e_{2}\right)$ for detecting the event $e_{2}$ is, for $\lambda$ odd,

$$
\begin{aligned}
& \left\langle N+\lambda\left|\hat{\rho}_{c}\right| N\right\rangle= \\
& \quad \frac{P_{0}\left(e_{2}\right)-P_{1}\left(e_{2}\right)+i\left[P_{1 / 2}\left(e_{2}\right)-P_{3 / 2}\left(e_{2}\right)\right]}{4 g_{N} g_{N+\lambda}^{*}}
\end{aligned}
$$

where

$$
g_{N} g_{N+\lambda}^{*}=i t^{2 N}(i r / 2)^{\lambda}\left|a_{0} a_{\lambda}^{*}\right|\left(\begin{array}{c}
\lambda \\
(\lambda-1) / 2
\end{array}\right)\left(\begin{array}{c}
N+\lambda \\
N
\end{array}\right)^{1 / 2}
$$

We note the number of phase settings required for each experiment for the odd $-\lambda$ case is about twice that required for the even- $\lambda$ case with a similar value of $\lambda$. The number of experiments needed for the odd- $\lambda$ case can, however, be reduced by a factor of two as follows. While measuring the probability for the event $e_{2}$, we can also measure the probability for another event $e_{3}$ $=[(\lambda-1) / 2,(\lambda+1) / 2, N]$. It is possible to show that the probability $P_{\beta}\left(e_{3}\right)$ is equal to $P_{\beta+1}\left(e_{2}\right)$. Thus we only need two experiments with different values of $\beta$ to obtain all four terms in the numerator of (2.20).

\section{SOME PRACTICAL CONSIDERATIONS}

To illustrate the viability of our proposal we shall, in this section, show the extent to which physical imperfections, such as a noisy local oscillator, detector inefficiency and dark counts, can be ignored or compensated for in a practical experiment.

A more general measurement scheme would have an arbitrarily mixed reference state at the input of mode $a$ in Fig. 1. That is $|\alpha\rangle_{a}$, with $\alpha=|\alpha| \exp (i \varphi)$ at the entry of BS2, would be replaced by a density operator $\exp \left(i \widehat{N}_{a} \varphi\right) \hat{\rho}_{a} \exp \left(-i \widehat{N}_{a} \varphi\right)$. This could represent, as a specific example, a noisy local oscillator. Following the derivation outlined above, it is straightforward to show the effect of this is to replace the term $\left|a_{0} a_{\lambda}^{*}\right|$ in equations (2.16) and (2.21) with the density matrix element $\left\langle 0\left|\hat{\rho}_{a}\right| \lambda\right\rangle$. This is interesting because it shows how the density matrix element $\left\langle N+\lambda\left|\hat{\rho}_{c}\right| N\right\rangle$ of the unknown field can be obtained directly from the density matrix element $\left\langle 0\left|\hat{\rho}_{a}\right| \lambda\right\rangle$ of the reference field. Thus, provided we know the noise characteristics of the reference state, we do not require it to be a noiseless coherent state, or indeed any particular pure state, to use it to find the density matrix
TABLE I: Truncated density matrix for a coherent state with a mean photon number of 0.5 .

\begin{tabular}{lllll}
\hline \hline 0.6065 & 0.4289 & 0.2145 & 0.0870 & 0.0336 \\
0.4289 & 0.3033 & 0.1517 & 0.0615 & 0.0238 \\
0.2145 & 0.1517 & 0.0759 & 0.0308 & 0.0119 \\
0.0870 & 0.0615 & 0.0308 & 0.0125 & 0.0048 \\
0.0336 & 0.0238 & 0.0119 & 0.0048 & 0.0019 \\
\hline \hline
\end{tabular}

TABLE II: Simulation of measured density matrix for a coherent state with a mean photon number of 0.5 and detector inefficiency $\eta=0.9$

\begin{tabular}{lllll}
\hline \hline 0.6592 & 0.4195 & 0.1888 & 0.0692 & 0.0220 \\
0.4195 & 0.2967 & 0.1335 & 0.0489 & 0.0161 \\
0.1888 & 0.1335 & 0.0668 & 0.0244 & 0.0081 \\
0.0692 & 0.0489 & 0.0244 & 0.0100 & 0.0033 \\
0.0220 & 0.0161 & 0.0081 & 0.0033 & 0.0013 \\
\hline \hline
\end{tabular}

elements of the unknown field. So we find in general that a noisy local oscillator can easily be used in the measurement scheme. A problem arises, however, if $\left\langle 0\left|\hat{\rho}_{a}\right| \lambda\right\rangle$ is vanishingly small in that the measured probabilities will coincide with rare events as indicated by (2.17) and (2.20). This is the case when phase diffusion in the local oscillator is prominent, effectively diagonalizing the density matrix $\hat{\rho}_{a}$ and removing all phase information. This can be avoided if both the reference field and the measured field, $\hat{\rho}_{c}$, are derived from a common source, a technique commonly exploited in experiments of this kind.

Another practical issue concerns the extent to which inefficient photodetectors degrade the reliability of the measured data. The effect of inefficient photodetectors is to make the measurement process uncertain. For a given detector efficiency $\eta$, the probability of detecting $n$ photons, $p_{n}(\eta)$, is related to the probability of detecting $m$ photons with a perfect detector, $p_{m}(1)$, by [19]

$$
p_{n}(\eta)=\sum_{m=0}^{\infty}\left(\begin{array}{c}
n+m \\
n
\end{array}\right) \eta^{n}(1-\eta)^{m} p_{n+m}(1)
$$

To illustrate what effect inefficient photodetectors have on the outcome of the experiment, some numerical calculations were performed for a low intensity coherent input state with a mean photon number of 0.5. An example of the results are summarized in Tables II and II, where the measured density matrix is displayed for a detector efficiency of $\eta=0.9$. The reference state used in each simulation was a coherent state with a mean photon number of $|\alpha|^{2}=0.5$. As expected, as the efficiency decreases the relative error in the individual matrix elements increases. Fortunately it is possible to invert equation (3.1) through a Bernoulli transformation and recover the exact probabilities from the detection statistics with sufficiently good 
detectors 19. This would allow accurate reconstruction of the density matrix.

In addition, for weak fields in the quantum regime, with sufficiently long gating times, dead times need not be significant. If dead times are significant, more sophisticated detection methods are required for photon number discrimination, such as replacing each detector with a multiport device such as described in [20].

So far we have not specified the value of $|\alpha|$ or $t / r$. The optimum values of these should maximize the denominators of $(2.17)$ and $(2.20)$, thereby avoiding quotients of small numbers. We find that the optimum value of $|\alpha|^{2}$ is $\lambda / 2$ and that of $(t / r)^{2}$ is $2 N / \lambda$. As these are optimum values only, they need not be changed for the measurement of each matrix element and a reasonable compromise value should suffice, for example, for weak fields where the spread of values of $N$ and $\lambda$ is not large.

While the method proposed in this paper can be used to measure any individual density matrix element, it is not necessary to perform the same number of cycling experiments as matrix elements to find the density matrix. The matrix elements $\left\langle N+\lambda\left|\hat{\rho}_{c}\right| N\right\rangle$ and their complex conjugates for all values of $N$ can be found from the same four cycling experiments. Also many phase settings can be used as parts of different cycling experiments, allowing further efficiencies. For example the setting $\varphi(\beta, j)$ $=\pi / 2$ can be used for $\beta=0, j=1, \lambda=4$ and $\beta=0$, $j=2, \lambda=8$ as well as for $\beta=1, j=0, \lambda=2$ and so on.

\section{CONCLUSION}

In this paper, we have extended the method of projection synthesis [21, in which a projector is synthesized by use of an exotic reference state, to a more general technique of operator synthesis in which an operator is synthesized by a linear combination of POM elements. This provides a nonrecursive method for measuring individual density matrix elements of a light field. Remarkably, the technique is reasonably simple, involving only two beam splitters and a reference field which can be in an easilyprepared coherent state. In particular, for states that can be represented in a finite dimensional Hilbert space, this technique appears simpler than the tomographic methods in that only a finite number of different measurements are required to ascertain the complete density matrix. We have shown how detector inefficiency can be allowed for and have considered the effect of noise in the local oscillator. We found that the local oscillator noise can be readily accounted for provided we know the correspond- ing mixed state description of the local oscillator. Interestingly, our method allows the density matrix elements of the unknown field to be obtained quite simply from the density matrix elements of a noisy local oscillator field, even when the unknown field is in a pure state.

\section{Acknowledgments}

DTP thanks the Australian Research Council for support.

\section{APPENDIX}

In this appendix we derive the general form of $|q\rangle_{c}$ defined by (2.7):

$$
|q\rangle_{c}={ }_{b}\left\langle 0\left|\widehat{R}_{1}^{\dagger}\left\langle\alpha\left|\widehat{R}_{2}^{\dagger}\right| n_{a}\right\rangle_{a}\right| n_{b}\right\rangle_{b}\left|n_{c}\right\rangle_{c} .
$$

With the unitary operator of a beam-splitter given by 18

$$
\widehat{R}=\exp \left[i \eta\left(\hat{a}^{\dagger} \hat{b}+\hat{b}^{\dagger} \hat{a}\right)\right],
$$

where $a$ and $b$ are the annihilation operators for the input field modes, it can be shown that the beam-splitter transforms the corresponding creation operators, $a^{\dagger}$ and $b^{\dagger}$, and the double mode vacuum according to

$$
\begin{aligned}
& \widehat{R}^{\dagger} \hat{a}^{\dagger} \widehat{R}=t \hat{a}^{\dagger}-i r \hat{b}^{\dagger} \\
& \widehat{R}^{\dagger} \hat{b}^{\dagger} \widehat{R}=t \hat{b}^{\dagger}-i r \hat{a}^{\dagger} \\
& \widehat{R}^{\dagger}|0\rangle_{b}|0\rangle_{c}=|0\rangle_{b}|0\rangle_{c}
\end{aligned}
$$

where $t$ and $r$ are the transmission and reflection coefficients of the beam-splitter. In the case of BS2, a $50 / 50$ beam-splitter, $t=r=1 / \sqrt{2}$. By writing $\left|n_{a}\right\rangle_{a}$ as $\left(\hat{a}^{\dagger n_{a}} / \sqrt{n_{a} !}\right)|0\rangle_{a}$, and similarly for $\left|n_{b}\right\rangle_{b}$, we obtain

$$
\widehat{R}_{2}^{\dagger}\left|n_{a}\right\rangle_{a}\left|n_{b}\right\rangle_{b}=\frac{\left(\hat{a}^{\dagger}-i \hat{b}^{\dagger}\right)^{n_{a}}\left(\hat{b}^{\dagger}-i \hat{a}^{\dagger}\right)^{n_{b}}}{2^{\left(n_{a}+n_{b}\right) / 2} \sqrt{n_{a} ! n_{b} !}}|0\rangle_{a}|0\rangle_{b}
$$

and thus

$$
\begin{aligned}
& { }_{a}\left\langle\alpha\left|\widehat{R}_{2}^{\dagger}\right| n_{a}\right\rangle_{a}\left|n_{b}\right\rangle_{b}|N\rangle_{c} \\
& =\frac{\left(\alpha^{*}-i \hat{b}^{\dagger}\right)^{n_{a}}\left(\hat{b}^{\dagger}-i \alpha^{*}\right)^{n_{b}}}{2^{\left(n_{a}+n_{b}\right) / 2} \exp \left(|\alpha|^{2} / 2\right) \sqrt{n_{a} ! n_{b} !}}|0\rangle_{b}|N\rangle_{c}
\end{aligned}
$$

Writing $|N\rangle_{c}$ as $\left(\hat{c}^{\dagger N} / \sqrt{N !}\right)|0\rangle_{c}$ and using an equivalent form of A.3 A.5, we obtain

$$
\begin{aligned}
& \widehat{R}_{1}^{\dagger}{ }_{a}\left\langle\alpha\left|\widehat{R}_{2}^{\dagger}\right| n_{a}\right\rangle_{a}\left|n_{b}\right\rangle_{b}|N\rangle_{c} \\
& \quad=\frac{\left[\alpha^{*}-i\left(t \hat{b}^{\dagger}-i r \hat{c}^{\dagger}\right)\right]^{n_{a}}\left[t \hat{b}^{\dagger}-i r \hat{c}^{\dagger}-i \alpha^{*}\right]^{n_{b}}\left(t \hat{c}^{\dagger}-i r \hat{b}^{\dagger}\right)^{N}}{2^{\left(n_{a}+n_{b}\right) / 2} \exp \left(|\alpha|^{2} / 2\right) \sqrt{n_{a} ! n_{b} ! N !}}|0\rangle_{b}|0\rangle_{c}
\end{aligned}
$$


where we have left the transmission and reflection coefficients of BS1 as $t$ and $r$. Finally, projecting onto the vacuum state in mode $b$ gives us

$$
\begin{aligned}
|q\rangle_{c} & =\frac{(-i)^{n_{b}} t^{N}\left[\alpha^{*}-r \hat{c}^{\dagger}\right]^{n_{a}}\left[\alpha^{*}+r \hat{c}^{\dagger}\right]^{n_{b}}}{2^{\left(n_{a}+n_{b}\right) / 2} \exp \left(|\alpha|^{2} / 2\right) \sqrt{n_{a} ! n_{b} !}}|N\rangle_{c} \\
& =\sum_{m=N}^{N+\lambda} q_{m}\left(n_{a}, n_{b}\right)|m\rangle_{c}
\end{aligned}
$$

where $\lambda=n_{a}+n_{b}$. The specific notation

$$
\begin{gathered}
f_{m}=q_{m}(\lambda / 2, \lambda / 2) \exp [i(N+\lambda-m) \varphi] \\
g_{m}=q_{m}[(\lambda+1) / 2,(\lambda-1) / 2] \exp [i(N+\lambda-m) \xi A .10)
\end{gathered}
$$

is used in the text. The explicit form of $q_{m}\left(n_{a}, n_{b}\right)$ is not actually needed. What is important is an expression for
$q_{N}\left(n_{a}, n_{b}\right) q_{N+\lambda}^{*}\left(n_{a}, n_{b}\right)$. This can be derived from (A.9) by evaluating $q_{N}\left(n_{a}, n_{b}\right)$ and $q_{N+\lambda}\left(n_{a}, n_{b}\right)$ separately to give

$$
\begin{aligned}
& q_{N}\left(n_{a}, n_{b}\right) q_{N+\lambda}^{*}\left(n_{a}, n_{b}\right) \\
& =(-1)^{n_{a}} a_{0} a_{\lambda}^{*} t^{2 N}(r / 2)^{\lambda}\left(\begin{array}{c}
\lambda \\
n_{a}
\end{array}\right)\left(\begin{array}{c}
N+\lambda \\
N
\end{array}\right)^{1 / 2} .
\end{aligned}
$$

where $a_{n}=\langle n \mid \alpha\rangle$. For a mixed reference state with density operator $\exp \left(i \widehat{N}_{a} \varphi\right) \hat{\rho}_{a} \exp \left(-i \widehat{N}_{a} \varphi\right)$ at the entry of BS2, $a_{0} a_{\lambda}^{*}$ in (A.11) is replaced by $\left\langle 0\left|\hat{\rho}_{a}\right| \lambda\right\rangle \exp (-i \lambda \varphi)$.

It is not difficult to see that the modulus of (A.11) is maximized when $n_{a}=\lambda / 2$ if $\lambda$ is even and when $n_{a}=$ $(\lambda \pm 1) / 2$ if $\lambda$ is odd. Thus the quotients in (2.17) and (2.20) will have optimum numerators and denominators for the values of $n_{a}$ and $n_{b}$ we have used in this paper.
[1] D. T. Smithey, M. Beck, M. G. Raymer, and A. Faridani, Phys. Rev. Lett 70, 1244 (1993); D. T. Smithey, M. Beck, J. Cooper, M. G. Raymer and A. Faridani, Phys. Scr. T48, 35 (1993).

[2] K. Vogel and H. Risken, Phys. Rev. A 40, R2847 (1989).

[3] K. Banaszek and K. Wódkiewicz, Phys. Rev. Lett. 76 4344 (1996).

[4] S. Wallentowitz and W. Vogel, Phys. Rev. A 53, 4528 (1996)

[5] J. H. Shapiro and S. S. Wagner, IEEE J. Quantum Electron. 20, 803 (1984)

[6] N. G. Walker and J. E. Carroll, Electron. Lett. , 981 (1984).

[7] J. W. Noh, A. Fougères, and L. Mandel, Phys. Rev. Lett. 67, 1426 (1991); Phys. Rev. A 45, 424 (1992).

[8] W. Vogel and J. Grabow, Phys. Rev. A 47, 4227 (1993).

[9] P. J. Bardroff, E. Mayr, and W. P. Schleich, Phys. Rev. A 51, 4963 (1995); P. J. Bardroff et al., Phys. Rev. A 53, 2736 (1996); L. G. Lutterbach and L. Davidovich, Phys. Rev. Lett. 78, 2547 (1997); M. S. Kim, G. Antesberger, C. T. Bodendorf, and H. Walther, Phys. Rev. A 58, R65 (1998).

[10] D. Leibfried et al., Phys. Rev. Lett. 77, 4281 (1996).

[11] D. G. Fischer and M. Freyberger, Opt. Commun. 159, 158 (1999).

[12] D.-G. Welsch, W. Vogel, and T. Opatrný, in Progress in Optics, edited by E. Wolf (North-Holland, Amsterdam, 1999), Vol. 39, p. 63, and references therein.

[13] U. Leonhardt, Measuring the Quantum State of Light (Cambridge University Press, Cambridge, 1997).

[14] M. S. Kim, G. Antesberger, C. T. Bodendorf, and H.
Walther, Phys. Rev. A 58, R65 (1998); D. T. Pegg, L. S. Phillips, and S. M. Barnett, J. Mod. Opt. 46, 981 (1999); D. T. Pegg and S. M. Barnett, J. Mod. Opt. 46, 1657 (1999); B. Rohwedder, L. Davidovich, and N. Zagury, Phys. Rev. A 60, 480 (1999); M. Mohmoudi, H. Tajalli, and M. S. Zubairy, J. Opt. B: Quantum Semiclass. Opt. 2, 315 (2000); A. Zucchetti, S. Wallentowitz, W. Vogel, and N. P. Bigelow, Phys. Rev. A 61, 011405 (2000); G. Nogues et al., Phys. Rev. A 62, 054101 (2000); M. F. Santos et al., Phys. Rev. A 63, 033813 (2001); C. J. Villas-Bôas, G. A. Prataviera, and M. H. Y. Moussa, Phys. Rev. A 64, 065801 (2001); S. B. Zheng, Commun. Theor. Phys. 36, 213 (2001).

[15] O. Steuernagel and J. A. Vaccaro, Phys. Rev. Lett. 75, 3201 (1995).

[16] A. I. Lvovsky, H. Hansen, T. Aichele, O. Benson, J. Mlynek, and S. Schiller, Phys. Rev. Lett. 87, 050402 (2001)

[17] C. W. Helstrom, Quantum Detection and Estimation Theory (Academic Press, New York, 1976).

[18] S. M. Barnett and P. M. Radmore, Methods in Theoretical Quantum Optics (Clarendon, Oxford, 1997).

[19] C. T. Lee, Phys. Rev. A 48, 2285 (1993); S. M. Barnett, L. S. Phillips and D. T. Pegg, Opt. Commun. 158, 45 (1998).

[20] H. Paul, P. Törmä, T. Kiss, and I. Jex, Phys. Rev. Lett. 76, 2464 (1996).

[21] S. M. Barnett and D. T. Pegg, Phys. Rev. Lett. 76, 4148 (1996). 